Open Access

\title{
Normal tissue complication probability modeling for cochlea constraints to avoid causing tinnitus after head-and-neck intensity-modulated radiation therapy
}

\author{
Tsair-Fwu Lee ${ }^{1,2}$, Shyh-An Yeh ${ }^{1,3,4^{*}}$, Pei-Ju Chao ${ }^{1,5}$, Liyun Chang ${ }^{4}$, Chien-Liang Chiu ${ }^{1,2}$, Hui-Min Ting ${ }^{1,5}$,
} Hung-Yu Wang ${ }^{1,2}$ and Yu-Jie Huang ${ }^{5}$

\begin{abstract}
Background: Radiation-induced tinnitus is a side effect of radiotherapy in the inner ear for cancers of the head and neck. Effective dose constraints for protecting the cochlea are under-reported. The aim of this study is to determine the cochlea dose limitation to avoid causing tinnitus after head-and-neck cancer (HNC) intensity-modulated radiation therapy (IMRT).
\end{abstract}

Methods: In total 211 patients with HNC were included; the side effects of radiotherapy were investigated for 422 inner ears in the cohort. Forty-nine of the four hundred and twenty-two samples (11.6\%) developed grade $2+$ tinnitus symptoms after IMRT, as diagnosed by a clinician. The Late Effects of Normal Tissues-Subjective, Objective, Management, Analytic (LENT-SOMA) criteria were used for tinnitus evaluation. The logistic and Lyman-Kutcher-Burman (LKB) normal tissue complication probability (NTCP) models were used for the analyses.

Results: The NTCP-fitted parameters were $T_{50}=46.31 \mathrm{~Gy}(95 \% \mathrm{Cl}, 41.46-52.50), \gamma_{50}=1.27(95 \% \mathrm{Cl}, 1.02-1.55)$, and $T D_{50}=46.52 \mathrm{~Gy}(95 \% \mathrm{Cl}, 41.91-53.43), m=0.35$ (95\% Cl, 0.30-0.42) for the logistic and LKB models, respectively. The suggested guideline $T_{20}$ for the tolerance dose to produce a $20 \%$ complication rate within a specific period of time was $T_{20}=33.62 \mathrm{~Gy}(95 \% \mathrm{Cl}, 30.15-38.27)$ (logistic) and $T_{20}=32.82 \mathrm{~Gy}(95 \% \mathrm{Cl}, 29.58-37.69)$ (LKB).

Conclusions: To maintain the incidence of grade $2+$ tinnitus toxicity $<20 \%$ in IMRT, we suggest that the mean dose to the cochlea should be <32 Gy. However, models should not be extrapolated to other patient populations without further verification and should first be confirmed before clinical implementation.

\section{Background}

Head-and-neck cancers (HNC) are some of the most serious malignancies worldwide. Radiation therapy (RT) is the mainstay of treatment, offered to almost $75 \%$ of all HNC patients with either curative or palliative intent. Toxicities associated with RT represent important clinical outcomes that can substantially reduce the patient's quality of life (QOL) and the ability of cancer patients to

\footnotetext{
* Correspondence: yeh5657@gmail.com

${ }^{1}$ Medical Physics and Informatics Laboratory of Electronics Engineering, National Kaohsiung University of Applied Sciences, 415, Chien Kung Road, San-Min District, Kaohsiung 80778 Taiwan, ROC

${ }^{3}$ Department of Radiation Oncology, E-Da hospital, No.1, Yida Road, Jiaosu Village, Yanchao District, Kaohsiung City 82445 Taiwan, ROC

Full list of author information is available at the end of the article
}

tolerate and complete the planned course of treatment [1]. During RT for HNC, the entire hearing apparatus and/or parts of the auditory system receive high doses of radiation during treatment, which can result in various radiation-induced damages to the external, middle, and inner ear [2,3]. Morbidities associated with the inner ear include a wide range of symptoms such as tinnitus, labyrinthitis, canal paresis, vertigo/balance problems, and sensori-neural hearing loss (SNHL) [2]. Hearing loss and neurological defects have been investigated extensively. However, radiation-induced tinnitus is underevaluated and under-reported.

The relationship between the dose of irradiation received by the cochlea and the degree of tinnitus 
toxicity experienced is well recognized, but poorly quantified [2, 4-6]. To our knowledge, no studies have described the normal tissue complication probability (NTCP) of the cochlea using tinnitus as the endpoint after radiation therapy. In addition, there is no Quantitative Analyses of Normal Tissue Effects in the Clinic (QUANTEC) guideline for the cochlea relating to the avoidance of tinnitus during intensity-modulated radiation therapy (IMRT). The NTCP parameters should, therefore, be determined to reveal the relationship between dose-response and radiation-induced tinnitus toxicity. Knowledge of the association between the dose distribution in the organs at risk and the probability of radiation-induced toxicity is becoming increasingly important during IMRT-planning procedures [4].

In this study, we used two common NTCP models, the logistic and the Lyman-Kutcher-Burman (LKB) models, to quantify the relationship between the incidence of tinnitus toxicity and the dose-response effects in the cochlea with the aim of identifying a specific dose relationship. The aim was to determine the best-fit parameters of these well-known and established NTCP models, and then identify the model that best describes the dose-response relationship between the cochlea and tinnitus after radiation therapy. The obtained results are likely to reveal a dose-response constraint for the cochlea that can reduce the incidence of tinnitus in radiation therapy using proper treatment planning.

\section{Methods}

\section{Study population}

In total 211 patients with $\mathrm{HNC}$ were included in this study; the side effects of radiotherapy were investigated for 422 inner ears in the cohort. All participants were treated using IMRT between June 2007 and December 2010. The characteristics of the samples are listed in Table 1. Any patients with problems related to the vestibulo-cochlear nerve were excluded from analysis because their hearing problems could be associated with pathologies of the auditory system [5, 7]. The Institutional Review Board of E-Da Hospital (EMRP-103-020) approved the study protocol, and all investigations were performed in accordance with the relevant guidelines and regulations.

A basic assumption, and a potential limitation, of the present study was that the two ears of each patient could be analyzed as independent variables. Patient age and observation time were, therefore, the same for both ears, and the dose and pre-therapeutic tinnitus levels could be expected to show some correlation [8]. Based on the suggestion by Honoré et al. [8], the maximum-likelihood estimate of variance components was used to assess the relative importance of variation between ears and among patients in the current study.
Table 1 Characteristics of patients with head and neck cancer

\begin{tabular}{|c|c|}
\hline & Value-x (\%) \\
\hline & $\mathrm{HNC}(\mathrm{n}=422)$ \\
\hline \multicolumn{2}{|l|}{ Age (y) } \\
\hline Mean & 50 \\
\hline Range & $26-73$ \\
\hline \multicolumn{2}{|l|}{ Gender ( $n)$} \\
\hline Male & $381(90.3 \%)$ \\
\hline Female & $41(9.7 \%)$ \\
\hline \multicolumn{2}{|l|}{ Tumor site } \\
\hline Larynx & 48 (11.4\%) \\
\hline Hypopharynx & $68(16.1 \%)$ \\
\hline Oropharynx & $54(12.8 \%)$ \\
\hline Oral cavity & $102(24.2 \%)$ \\
\hline Nasopharyngeal carcinoma & $150(35.5 \%)$ \\
\hline \multicolumn{2}{|l|}{ AJCC stage } \\
\hline । & 0 \\
\hline$\|$ & $34(8.1 \%)$ \\
\hline III & $95(22.5 \%)$ \\
\hline IV & $293(69.4 \%)$ \\
\hline \multicolumn{2}{|l|}{ Cochlea mean dose } \\
\hline $1-10$ & $43(10.2 \%)$ \\
\hline $10-20$ & $118(28 \%)$ \\
\hline $20-30$ & $139(32.9 \%)$ \\
\hline $30-40$ & $101(23.9 \%)$ \\
\hline $40-50$ & $15(3.6 \%)$ \\
\hline $50-60$ & $6(1.4 \%)$ \\
\hline \multicolumn{2}{|l|}{ Tinnitus (grade $2+$ ) } \\
\hline Yes & 49 (11.6\%) \\
\hline No & $373(88.4 \%)$ \\
\hline Cochlea mean dose Gy (range) & $23.72(1.73-58.83)$ \\
\hline Without grade $2+$ Tinnitus & $22.44(1.73-50.26)$ \\
\hline With grade $2+$ Tinnitus & $33.46(8.72-58.83)$ \\
\hline \multicolumn{2}{|l|}{ Chemotherapy } \\
\hline Yes & $150(35.5 \%)$ \\
\hline No & $272(64.5 \%)$ \\
\hline
\end{tabular}

Abbreviation: AJCC American Joint Committee on Cancer, HNC head and neck cancer

\section{IMRT}

Each patient's head and shoulders were immobilized using a commercially available thermoplastic mask and/ or an individually customized bite block. Computed tomography (CT) images (2.5-mm slice thickness, $512 \times$ 512 pixels/slice) were acquired from the top of the vertex to the level of the carina (simulation CT; LightSpeed RT16, GE Medical Systems, Waukesha, WI, USA). All patients were treated using IMRT as previously described in detail [9]. The mean dose to the cochlea was 
kept as low as possible while still achieving the desired clinical target volume coverage. The dose distributions were calculated and dose-volume histograms (DVHs) were generated separately for each cochlea. Two IMRT techniques were used: simultaneous integrated boost (SIB), and sequential mode (SQM). Details regarding the prescribed dose and fractions for the SIB and SQM techniques can be found in previous studies [9, 10]. IMRT was delivered using the computer-controlled auto-sequencing segment or the dynamic multileaf collimator of a linear accelerator (Elekta Precise, Elekta, Crawley, UK) according to methods reported elsewhere [11]. The prescribed doses were 66.0-77.4 Gy (median, 70.0 Gy) to the macroscopic tumor (planning target volume 1 [PTV1]), 54.0-66.0 Gy (median, 61.2 Gy) to the resected tumor bed (PTV2), and 41.4-54.0 Gy (median, 50.4 Gy) to the subclinical disease area (PTV3). These were administered at 1.6-2.12 Gy per fraction using SIB, and 1.8-2.0 Gy per fraction using SQM with five fractions per week.

According to the Radiation Therapy Oncology Group 0225 and 0615 trials, the planning objectives for PTVs were a minimum dose $>95 \%$, and no more than $5 \%$ of any PTV1 received $\geq 110 \%$ of the prescribed dose. The mean dose constraints for the parotid gland were a mean dose $\leq 26$ Gy or $V_{30 G y} \leq 50 \%$. For the oral cavity excluding the PTV; a mean dose $\leq 40$ Gy was used. No mean dose constraints for the cochlea to avoid tinnitus have been reported previously. In most cases, the mean dose constraint to the cochlea was limited to $<45 \mathrm{~Gy}$. If the cochlea was adjacent to or inside the PTV1 or PTV2, the mean dose to the cochlea could be higher so as not to sacrifice the PTV coverage. The DVH values were calculated for each cochlea in all patients. All data were based on the DVHs obtained using Pinnacle ${ }^{3_{0}}$ (Philips, Fitchburg, WI, USA) with a bin size resolution of $0.01 \mathrm{~Gy}$. The resolution of the dose calculation was $2.5 \mathrm{~mm}$ for all IMRT plans.

\section{Evaluation of tinnitus}

After the completion of radiotherapy, patients were examined at 4-week intervals until their acute radiation-related complications subsided. Patients were subsequently followed-up every 2 months for the first year, and every 3 months thereafter. The evaluations performed at each follow-up visit included a medical history and physical examination. Hematology and biochemistry profiles, chest radiographs, sonography of the abdomen, a bone scan, and a CT scan of the head and neck were performed at least annually, and were checked whenever there was any clinical indication. The median follow-up period before the first detection of grade 2 tinnitus was 23 months (range: 19-37 months). The median followup period for the entire cohort of patients was 51 months (range: $36-77$ ).
According to the Late Effects of Normal Tissues-Subjective, Objective, Management, Analytic (LENT-SOMA) criteria for tinnitus evaluation, the following five-point scale was used: 0 , none; 1 , occasional; 2 , intermittent; 3 , persistent; and 4, refractory [12]. Generally, grade 1 toxicities are radiographic findings of negligible clinical consequence that are rarely scored in reports of RT-induced toxicity. Grade 2 to 4 toxicities generally reflect moderate, severe, or irreversible functional damage, respectively [13].

The threshold of grade 2+ tinnitus was used as an endpoint for toxicity. The LENT-SOMA criteria for tinnitus did not define the minimum number of observations used to define the grade of toxicity. In the current study, a patient with tinnitus $\geq$ grade 2 on at least two observations was enrolled. Although this was a retrospective study, we assessed the occurrence of tinnitus after radiotherapy over a long period of time; the median follow-up period was 23 months. In general, a minimum follow-up period of 12 months was required before a patient could be recorded. Since there is a potential for some patients with otorrhea to develop otitis media within the first few months following radiotherapy, which would lead to temporary hearing impairment or tinnitus, such patients would be observed until the otorrhea had subsided. Any tinnitus persisting after the cessation of otorrhea would then be noted. Therefore, the hearing of all patients was evaluated carefully (including the presence of tinnitus) before beginning radiotherapy, and the history of tinnitus was reviewed to rule out symptoms that were not induced by radiation.

\section{Dose-response modeling}

Two commonly used NTCP models, logistic and LKB [14-21], were used to quantify the relationship between the incidence of tinnitus toxicity and the dose-response effects on the cochlea.

A logistic model was used to fit the dose-response for the incidence of grade $2+$ tinnitus as a function of the mean dose to the cochlea according to the following formula:

$$
N T C P=\frac{\exp \left(4 \gamma_{50}\left(\frac{M D}{T D_{50}}-1\right)\right)}{1+\exp \left(4 \gamma_{50}\left(\frac{M D}{T D_{50}}-1\right)\right)}
$$

where $M D$ is the mean dose to the cochlea; $T D_{50}$ is the mean dose predicting a $50 \%$ risk of complications; and $\gamma_{50}$ is the normalized slope of the dose-response curve; i.e., the change in NTCP per $1 \%$ change in dose [22].

The family of Lyman-Kutcher-Burman (LKB) models is the most widely used phenomenological approach [14-21]. The LKB model is described by three parameters: $n, m$, and $T D_{50}$. According to this model, NTCP is characterized by three equations: 


$$
\begin{aligned}
& N T C P=\frac{1}{\sqrt{2 \pi}} \int_{-\infty}^{t} \exp \left(-x^{2} / 2\right) d x \\
& t=\frac{D_{\text {eff }}-T D_{50}}{m \cdot T D_{50}} \\
& D_{\text {eff }}=\left(\sum_{i=1}^{N} v_{i} \cdot D_{i}^{1 / n}\right)^{n}
\end{aligned}
$$

where $D_{\text {eff }}$ is the dose given to the entire volume ( $D_{\text {eff }}$ is sometimes referred to as the equivalent uniform dose, EUD), $n$ is a parameter that considers the volume effect, and $v_{i}$ is the volume of the dose bin that corresponds to dose $D_{i}$ in the differential DVH.

The parameter $m$ is a unitless model parameter for describing the slope of the dose-response curve. In this study, $n$ was set to 1 . Therefore, $D_{\text {eff }}$ reduces to an expression for the mean organ dose (MD). In such special cases, the LKB model can be simplified to

$$
N T C P=\frac{1}{\sqrt{2 \pi}} \int_{-\infty}^{t} \exp \left(-x^{2} / 2\right) d x, \text { where } t=\frac{M D-T D_{50}}{m \cdot T D_{50}}
$$

The best-fit values for $T D_{50}, \gamma_{50}$, and $m$ were identified using maximum-likelihood (ML) analysis, and the $95 \%$ confidence intervals (CIs) were calculated using the profile-likelihood method while the parameters were fixed at the ML estimate [23]. The CIs were calculated by fixing one parameter at its best-fit value and allowing the parameter of interest to vary. The $95 \%$ lower and upper confidence bounds were displaced downward from the ML peak by a distance determined from the chi-square distribution, $\chi^{2}(0.05,1) / 2=1.92[24,25]$. In addition, the guideline $T D_{20}$ was suggested as the tolerance dose that produced a $20 \%$ complication rate within a specific period of time. All calculations were performed using the Matlab software (R2010; MathWorks, Natick, MA, USA). Although the logistic and the Lyman model yield similar NTCP parameters, they are not precisely equivalent. However, part of the aims of this study was to verify the assertion that the Lyman and logistic models are broadly similar for the same dataset.

\section{Performance evaluation}

The model performance was measured using a variety of validation tools [26-28]. The overall performance was expressed as a scaled Brier score and checked by an Omnibus test. The scaled Brier score evaluates the differences between actual outcome and predictions, and a scaled Brier score of 1 would provide optimal agreement between predictions and actual outcomes. Model performance was also verified using the area under the receiver operating characteristic curves (AUC), and by calculating the discrimination slope, which was defined as the absolute difference between the mean predicted
NTCP-values of patients with and without the evaluated endpoint [29]. The goodness of fit was further quantified in terms of calibration; i.e., the agreement between the predicted and observed outcomes in the dataset, whereas the Hosmer-Lemeshow test was used to test the agreement between the expected and observed outcomes. A Hosmer-Lemeshow test value $>0.05$ would show agreement between expected and observed outcomes; however, a value $>0.15$ is recommended. The calibration curves were plotted to show the relationship between predicted risk and real outcome, and the curved slope of 1 showed a perfect match. The size of bin used was $10 \mathrm{~Gy}$.

Akaike's information criterion (AIC) was used to rank the accepted models. Models with smaller AIC values were considered to provide a better fit to the data than were models with larger AIC values [22]. Delta AIC $(\triangle \mathrm{AIC})$ is a measure of each model relative to the best model. As a rule of thumb, $\triangle \mathrm{AIC}=\mathrm{AICi}-$ minAIC, whereas a $\triangle \mathrm{AIC}>10$ indicates that the model is very unlikely; values of 3-10 indicate that the model has considerably less support; values $<2$ suggest substantial evidence for the model and can be considered to be similar to the best model [30-32].

The negative predictive value (NPV) was also calculated, which described the rate of avoiding tinnitus when the guidelines were fulfilled based on dose-volume data. For example, NPV-TD 20 is the fraction of all patients who had a dose below $T D_{20}$ and who did not have toxicity. A high NPV supports the validity of a suggested guideline $\left(T D_{20}\right)$, although plans near the guideline threshold are less risky than are those distinct from the threshold $\left(T D_{50}\right)$. These analyses were performed for both $T D_{50}$ and $T D_{20}$ criteria. All statistical analyses were performed using the SPSS 19.0 software (SPSS, Chicago, IL, USA).

\section{Results}

Three patients who already suffered from problems related to the vestibulo-cochlear nerve were excluded, leaving 422 samples from 211 patients to be analyzed. Forty-nine of the four hundred and twenty-two samples (11.6\%) developed grade 2+ tinnitus symptoms determined by a clinician to be caused by IMRT. The data regarding patient outcome are summarized in Table 1. The maximum-likelihood estimate of variance components revealed that there was no significant variation among the patient symptoms. This is consistent with the observations of Honoré et al., and therefore the two ears of a patient could be used as independent variables of tinnitus. Average values of cochlea mean doses for patients with and without tinnitus were 33.46 Gy and 22.44 Gy, respectively. Univariate analysis was performed, which revealed a significant relationship between grade $2+$ tinnitus and the mean dose to the cochlea $(p<0.001)$. 
The fitted dose-response curves (logistic and LKB NTCP models) for the incidence of grade $2+$ tinnitus in the HNC patient cohort are shown in Fig. 1a, b. The NTCP-fitted parameters for the logistic and LKB models were $T D_{50}=46.31$ Gy (95\% CI, 41.46-52.50), $\gamma_{50}=1.27$ (95\% CI, 1.02-1.55), and $T D_{50}=46.52 \mathrm{~Gy}$ (95\% CI, 41.91-53.43), $m=0.35$ (95\% CI, 0.30-0.42), respectively (Table 2 ). A simple formula can be used for convenience; i.e., $\gamma_{50} \approx 0.4 / \mathrm{m}$. The odds ratio for the logistic model was 1.117 (95\% CI, 1.078-1.157). The suggested guideline $T D_{20}$ for the tolerance dose to produce a $20 \%$ complication rate within a specific period of time was $T D_{20}=33.62$ Gy (95\% CI, 30.15-38.27) (logistic) and $T D_{20}=32.82$ Gy (95\% CI, 29.58-37.69) (LKB).

The overall performance and calibration of the NTCP models for grade $2+$ tinnitus toxicity according to the

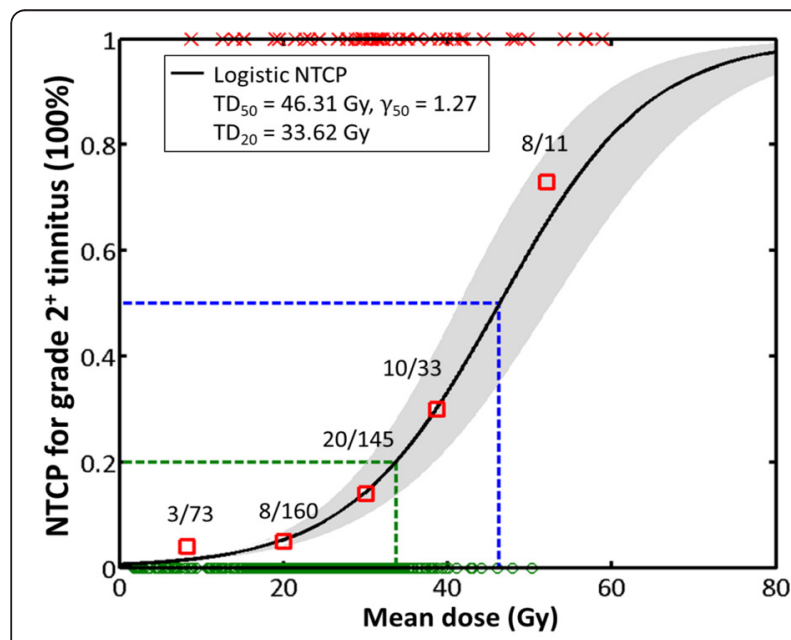

(a)

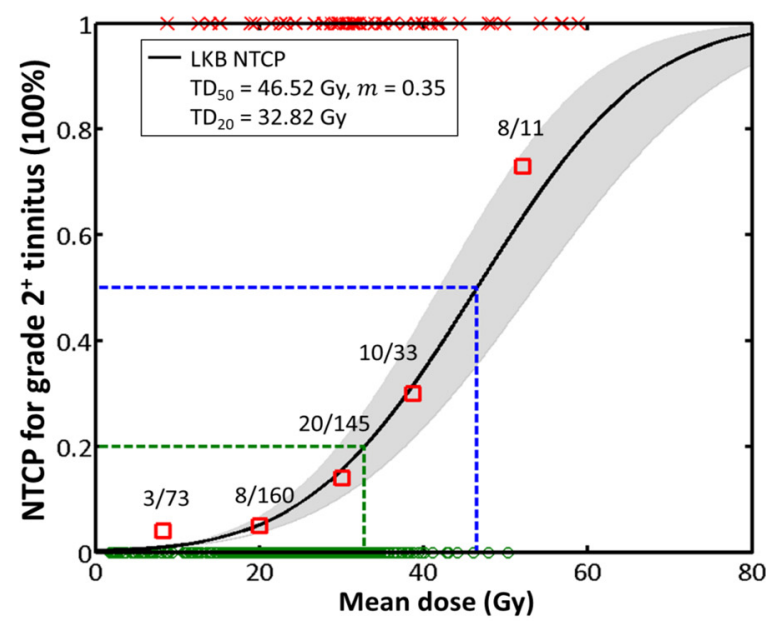

(b)

Fig. 1 The fitted dose-response curves of the a logistic and $\mathbf{b} L K B$ NTCP models for the incidence of grade $2+$ tinnitus. NTCP normal tissue complication probability, LKB Lyman-Kutcher-Burman scaled Brier score, AUC, Hosmer-Lemeshow tests, and the calibration slope were satisfactory and within the expected ranges (Table 3). The AUC for the optimal model was 0.76 (95\% CI, 0.69-0.84) and 0.76 (95\% CI, 0.69-0.84) for the logistic and LKB NTCP models, respectively. The NPVs are provided in Table 3, and data revealed that there were very low rates of grade $2+$ tinnitus. Smaller AIC values are preferable when comparing two models. However, from the point of view of $\triangle \mathrm{AIC}$, the logistic model is comparable with the LKB model for fitting this study dataset $(\triangle \mathrm{AIC}=1.69)$. Finally, the calibration slope was $\geq 0.99$ for both models, which revealed significant agreement between the predicted risk and observed outcome using both NTCP models (Fig. 2). The circles in Fig. 2 represent groups of patients with a specific mean calculated probability. The corrected NTCP is the trend line between the data points compared with the reference line, which indicates perfect calibration between predicted risk and real outcome. Overall, these data lead to the suggestion that the mean dose to the cochlea should be maintained at $<32$ Gy to keep the incidence of grade $2+$ tinnitus toxicity at $<20 \%$ in IMRT.

\section{Discussion}

In this study, the data revealed that the occurrence of grade 2+ tinnitus after IMRT was $11.6 \%$. Previous studies have reported a wide range of incidences of radiation-induced tinnitus. For example, van der Putten et al. reported a $12 \%$ incidence with parotid gland tumors that were treated using local or locoregional unilateral postoperative radiotherapy [4], whereas Tuan et al. described an incidence of $12 \%$ in nasopharyngeal cancer using conventional radiotherapy [5]. In addition, Bakhshandeh reported an occurrence of $13.5 \%$ in head and neck cancer treated using conventional radiotherapy [22], whereas Litre reported a much higher incidence of $64 \%$ after the use of fractionated stereotactic radiotherapy to treat acoustic neuromas [6]. The reasons for these differences could be related to the different cancers, treatment techniques, prescription doses resulting in a difference in dose to the cochlea, or evaluation grading schemes used.

There are no current standard dose-response constraints for the cochlea because no specific NTCP values have been reported to be associated with an increased incidence of tinnitus toxicity. Quantitative Analyses of Normal Tissue Effects in the Clinic (QUANTEC), a tool resulting from recent concerted efforts among the radiotherapy community, includes reviews and summaries of normal tissue toxicity datasets and suggested dose-volume treatment planning guidelines to reduce the incidence of side effects [33]. However, there is no QUANTEC guideline for the cochlea to avoid tinnitus during IMRT. QUANTEC recommends a $T D_{25} \leq 14$ Gy for SNHL for 
Table 2 Normal tissue complication probability fitted parameters

\begin{tabular}{lllr}
\hline NTCP model & $T D_{50}$ Gy $(95 \%$ Cl) & $\gamma_{50}$ or $m(95 \%$ Cl) & $T D_{20}$ Gy $(95 \%$ Cl) \\
\hline Logistic & $46.31(41.46-52.50)$ & $1.27(1.02-1.55)$ & $33.62(30.15-38.27)$ \\
LKB & $46.52(41.91-53.43)$ & $0.35(0.30-0.42)$ & $32.82(29.58-37.69)$
\end{tabular}

Abbreviation: $C l$ confidence interval, NTCP normal tissue complication probability, $L K B$ Lyman-Kutcher-Burman, $T D_{50}$ the dose predicting a $50 \%$ risk of complications, $T D_{20}$ the dose predicting a $20 \%$ risk of complications, $m$ a unitless LKB model parameter for describing the slope of the dose-response curve, $\gamma_{50}$ a Logistic model parameter for normalized slope of the dose-response curve

The Cls were calculated by fixing one parameter at its best-fit value and allowing the other parameter to vary

hearing at $4 \mathrm{kHz}$ during the stereotactic radiosurgery for HNCs and vestibular schwannomas, and a $T D_{30} \leq 45 \mathrm{~Gy}$ for three-dimensional conformal radiotherapy (3D-CRT) [34]. In the current study, we suggested that the mean dose to the cochlea should be maintained at $<32$ Gy to maintain the incidence of grade $2+$ tinnitus toxicity at $<20 \%\left(T D_{20}\right)$ during IMRT.

The most serious radiation-induced complication for the inner ear is SNHL. Published studies show consistently that post-irradiation SNHL occurs in approximately $30 \%$ of patients treated with definitive radiation using fields including the inner ear (cochlea) [12]. Although there is no agreement regarding cochlear radiation dose constraints, the minimum cochlear radiation dose reported as a risk factor for SNHL previously was $45-70$ Gy $[4,8,35,36]$. In the current study, we identified mean dose constraints of $T D_{50}$ and $T D_{20}$ for tinnitus; the mean dose to the cochlea must be maintained below $46 \mathrm{~Gy}$ if a $50 \%$ or less probability of complication is desired. If the probability must be maintained below $20 \%$ then the mean dose to the cochlea should be maintained below 32 Gy. These could be particularly advantageous for the treatment of $\mathrm{HNC}$, when large regions require treatment and the toxicity that affects critical normal structures can have a profound impact on the patient function and quality of life [37]. $T D_{50}$ and $T D_{20}$ values can provide guidance for setting dose constraints and predicting the risk of grade $2+$ tinnitus during

Table 3 System performance evaluation

\begin{tabular}{|c|c|c|}
\hline & Logistic NTCP & LKB NTCP \\
\hline AUC & $0.76(0.69-0.84)$ & $0.76(0.69-0.84)$ \\
\hline Brier (scaled) & 0.16 & 0.16 \\
\hline Omnibus & $P<0.001$ & $P<0.001$ \\
\hline $\mathrm{HL}$ test ( $p$-value) & 0.82 & 0.43 \\
\hline NPV-TD 50 & 0.90 & 0.90 \\
\hline NPV-TD 20 & 0.92 & 0.92 \\
\hline $\mathrm{AIC}$ & 257.79 & 259.48 \\
\hline
\end{tabular}

Abbreviation: NTCP normal tissue complication probability, LKB Lyman-KutcherBurman, NPV Negative predictive value, AUC Area under the receiver operating characteristic curve, HL Hosmer-Lemeshow test, NPV negative predictive value, $T D_{50}$ the dose predicting a $50 \%$ risk of complications, $T D_{20}$ the dose predicting a $20 \%$ risk of complications, AIC Akaike's information criterion Bin sizes used for Hosmer-Lemeshow test were 15<, 15-25, 25-35, 35-45, >45 Gy (five bins)

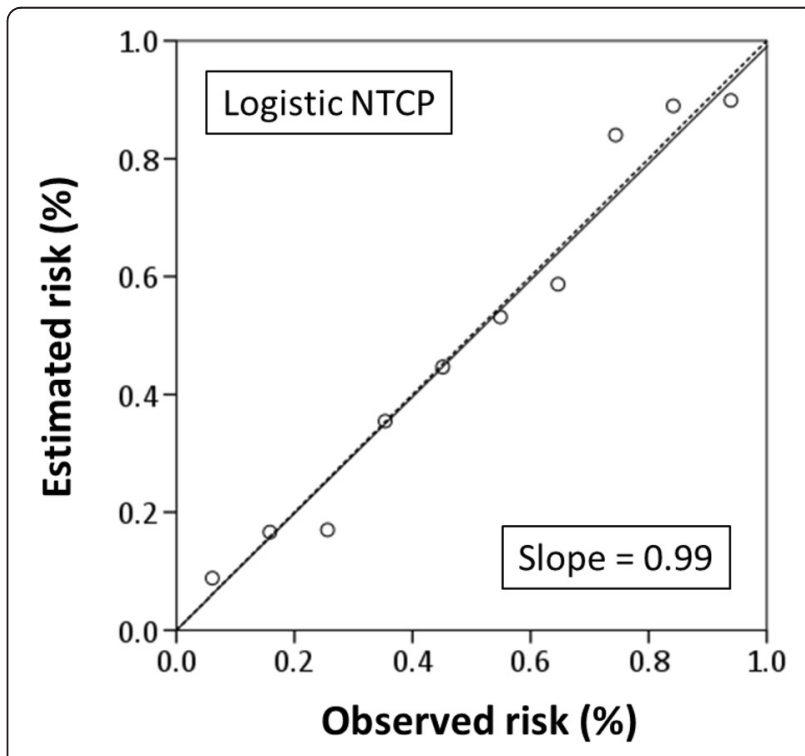

(a)

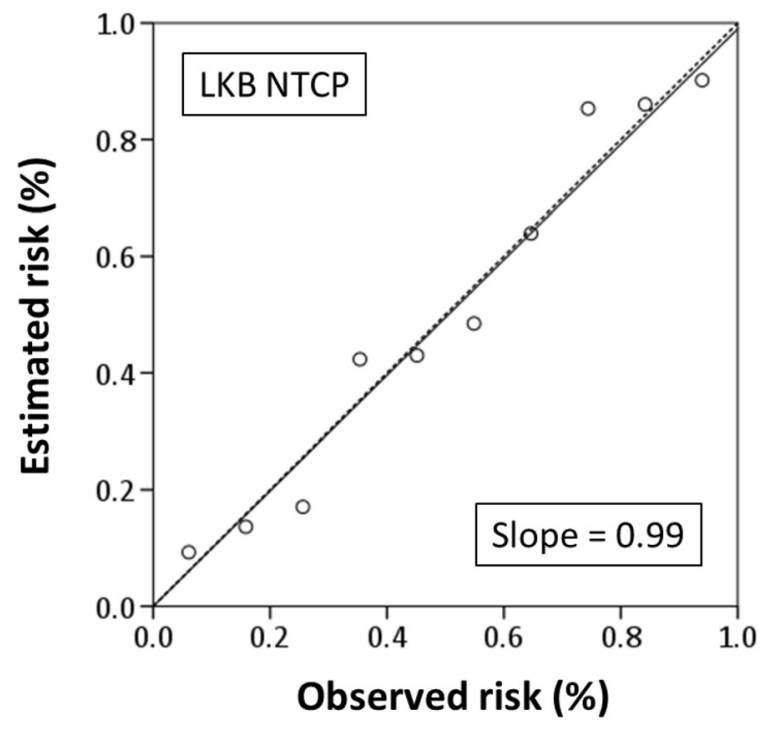

(b)

Fig. 2 Calibration curve of the predictive models for grade 2+ tinnitus using a logistic and $\mathbf{b}$ LKB NTCP. The plots show the relationship between estimated risk and real outcome. NTCP normal tissue complication probability, LKB Lyman-Kutcher-Burman. Bin size $=6 \mathrm{~Gy}$ 
treatment planning. The dose-response model described in the current study could be useful for comparing the grade 2+ tinnitus rates among different dose-fractionation schedules using IMRT for HNC.

In the current study, the dose response of the cochlea for grade $2+$ tinnitus after HNC radiation therapy was used for NTCP modeling using logistic or LKB models. A goal of the current study was the retrospective enrollment of clinical data and its fitting to two different NTCP models, even though it is known that there is no difference in the fitting of logistic NTCP or LKB NTCP to the same dataset. Based on the observation that NTCP models provide a similar description of the data and the phenomenological nature of the models, the simplest model from a computational point of view could be suggested; i.e., the logistic NTCP model. The logistic NTCP model also has a smaller AIC value. The results presented in the current study suggest that the clinical data could be fitted to two NTCP models to obtain similar $T D_{50}$ values.

In the current study, we defined constraints for the mean dose to the cochlea instead of the dose-volume relationship. A dose-volume analysis is unsuitable for the cochlea because of its small volume and the limitations associated with its delineation [34]. Several studies have attempted to relate the mean or median cochlear dose to persistent hearing impairment $[8,34,38]$. All of these analyses focused on the mean dose given the small anatomical size of the cochlea. We used the maximal, mean, and minimal doses to the cochlea as the dose metrics in the NTCP models, and found that the mean dose had better correlation than did the other doses. Therefore, we selected the mean dose for use in the NTCP modeling and subsequent analyses.

Despite our observations, a large individual study sample is needed to demonstrate the independent association of these NTCP models and the risk of grade 2+ tinnitus toxicity. Moreover, treatment methods might differ among nations and institutions, and differences in radiation modalities might yield different types and levels of tinnitus toxicity. The risk of the cochlea might also be influenced by the techniques used for treatment or factors other than the dose, such as baseline patient risk factors or the co-irradiation of other organs. Therefore, these parameters should be investigated further.

\section{Conclusion}

The dose-response limitation for the cochlea to maintain the incidence of grade $2+$ tinnitus toxicity below $20 \%$ in IMRT is that the mean dose to the cochlea should be maintained below 32 Gy. However, NTCP fits can vary based on a variety of factors, such as patient characteristics, treatment technique, follow up time, etc. Models should not be extrapolated to other patient populations without further verification and should be confirmed before clinical implementation.

\begin{abstract}
Abbreviations
HNC: Head and neck cancer; IMRT: Intensity-modulated radiation therapy; LKB: Lyman-Kutcher-Burman; NTCP: Normal tissue complication probability; AJCC: American Joint Committee on Cancer; Cl: Confidence interval; $T D_{50}$ : the dose predicting a $50 \%$ risk of complications; $T D_{20}$ : the dose predicting a $20 \%$ risk of complications; $m$ : a unitless LKB model parameter for describing the slope of the dose-response curve; $\gamma_{50}$ : a Logistic model parameter for normalized slope of the dose-response curve; RT: Radiation therapy; SNHL: Sensori-neural hearing loss; CT: Computed tomography; DVH: Dose-volume histograms; SIB: Simultaneous integrated boost; SQM: Sequential mode; PTV: Planning target volume; LENT-SOMA: the Late Effects of Normal Tissues-Subjective, Objective, Management, Analytic; NPV: Negative predictive value; AUC: Area under the receiver operating characteristic curve; HL: Hosmer-Lemeshow test; AIC: Akaike's information criterion.
\end{abstract}

\section{Competing interests}

The authors have declared that no competing interests exist.

\section{Authors' contributions}

TFL: had given valuable advices on the proceeding of this work, designed the concept and the experiment method of the research, wrote the manuscript and revised the manuscript. SAY: supported the characterization of the samples, analyzed the data and wrote the manuscript and revised the manuscript. PJC, LYC \& HMT: had given valuable advices and technical supports on data collection and analysis. HYW and CLC: technical supports on revised manuscript. YJH: had provided valuable suggestions and revised the manuscript. All authors read and approved the final manuscript.

\section{Acknowledgements}

We thank Shih-Sian Guo and Yi-Ling Chen for technical supports. This study was supported financially, in part, by grants from the Ministry of Science and Technology (MOST) of the Executive Yuan of the Republic of China, (NSC-101-2221-E-151-007-MY3 and MOST 104-2221-E-151-017). The funders had no role in study design, data collection and analysis, decision to publish, or preparation of the manuscript. Parts of our results were presented in an abstract form at Global Biotechnology Congress 2015, Boston, USA.

\section{Author details}

${ }^{1}$ Medical Physics and Informatics Laboratory of Electronics Engineering, National Kaohsiung University of Applied Sciences, 415, Chien Kung Road, San-Min District, Kaohsiung 80778 Taiwan, ROC. ${ }^{2}$ Institute of Clinical Medicine, Kaohsiung Medical University, Kaohsiung 807 Taiwan, ROC. ${ }^{3}$ Department of Radiation Oncology, E-Da hospital, No.1, Yida Road, Jiaosu Village, Yanchao District, Kaohsiung City 82445 Taiwan, ROC. ${ }^{4}$ Department of Medical Imaging and Radiological Sciences, I-Shou University, Kaohsiung 840 Taiwan, ROC. ${ }^{5}$ Department of Radiation Oncology, Kaohsiung Chang Gung Memorial Hospital and Chang Gung University College of Medicine, Kaohsiung 83305 Taiwan, ROC.

Received: 22 March 2015 Accepted: 4 September 2015

Published online: 17 September 2015

\section{References}

1. Ratko T, Douglas G, de Souza J, Belinson S, Aronson N. Radiotherapy treatments for head and neck cancer update. 2014.

2. Bhandare N, Antonelli PJ, Morris CG, Malayapa RS, Mendenhall WM. Ototoxicity after radiotherapy for head and neck tumors. Int J Radiat Oncol Biol Phys. 2007;67:469-79.

3. Borsanyi SJ, Blanchard CL. lonizing radiation and the ear. JAMA. 1962;181:958-61.

4. van der Putten L, de Bree R, Plukker JT, Langendijk JA, Smits C, Burlage FR, et al. Permanent unilateral hearing loss after radiotherapy for parotid gland tumors. Head Neck. 2006;28:902-8.

5. Tuan JKL, Ha TC, Ong WS, Siow TR, Tham IWK, Yap SP, et al. Late toxicities after conventional radiation therapy alone for nasopharyngeal carcinoma. Radiother Oncol. 2012;104:305-11. 
6. Litre F, Rousseaux P, Jovenin N, Bazin A, Peruzzi P, Wdowczyk D, et al. Fractionated stereotactic radiotherapy for acoustic neuromas: a prospective monocenter study of about 158 cases. Radiother Oncol. 2013;106:169-74.

7. Low WK, Toh ST, Wee J, Fook-Chong SM, Wang DY. Sensorineural hearing loss after radiotherapy and chemoradiotherapy: a single, blinded, randomized study. J Clin Oncol. 2006;24:1904-9.

8. Honoré HB, Bentzen SM, Møller K, Grau C. Sensori-neural hearing loss after radiotherapy for nasopharyngeal carcinoma: individualized risk estimation. Radiother Oncol. 2002;65:9-16.

9. Lee T-F, Fang F-M. Quantitative analysis of normal tissue effects in the clinic (QUANTEC) guideline validation using quality of life questionnaire datasets for parotid gland constraints to avoid causing xerostomia during head-andneck radiotherapy. Radiother Oncol. 2013;106:352-8.

10. Lee T-F, Ting H-M, Chao P-J, Fang F-M. Dual Arc volumetric-modulated Arc radiotherapy (VMAT) of nasopharyngeal carcinomas: a simultaneous integrated boost treatment plan comparison with intensity-modulated radiotherapies and single arc VMAT. Clin Oncol. 2012;24:196-207.

11. Chen W-C, Hwang T-Z, Wang W-H, Lu C-H, Chen C-C, Chen C-M, et al. Comparison between conventional and intensity-modulated post-operative radiotherapy for stage III and IV oral cavity cancer in terms of treatment results and toxicity. Oral Oncol. 2009;45:505-10.

12. Jereczek-Fossa BA, Zarowski A, Milani F, Orecchia R. Radiotherapy-induced ear toxicity. Cancer Treat Rev. 2003;29:417-30.

13. Pavy J-J, Denekamp J, Letschert J, Littbrand B, Mornex F, Bernier J, et al. Late effects toxicity scoring: the SOMA scale. Int J Radiat Oncol Biol Phys. 1995;31:1043-7.

14. Dijkema T, Raaijmakers CPJ, Ten Haken RK, Roesink JM, Braam PM, Houweling AC, et al. Parotid gland function after radiotherapy: the combined Michigan and Utrecht experience. Int J Radiat Oncol Biol Phys. 2010;78:449-53.

15. Lyman JT. Complication probability as assessed from dose-volume histograms. Radiat Res. 1985;104:S13-9.

16. Lyman JT, Wolbarst AB. Optimization of radiation therapy, III: a method of assessing complication probabilities from dose-volume histograms. Int J Radiat Oncol Biol Phys. 1987;13:103-9.

17. Kutcher GJ, Burman C. Calculation of complication probability factors for non-uniform normal tissue irradiation: the effective volume method gerald. Int J Radiat Oncol Biol Phys. 1989;16:1623-30.

18. Kutcher G, Burman C, Brewster L, Goitein M, Mohan R. Histogram reduction method for calculating complication probabilities for three-dimensional treatment planning evaluations. Int J Radiat Oncol Biol Phys. 1991;21:137-46.

19. Niemierko A, Goitein M. Calculation of normal tissue complication probability and dose-volume histogram reduction schemes for tissues with a critical element architecture. Radiother Oncol. 1991;20:166-76.

20. Söhn M, Yan D, Liang J, Meldolesi E, Vargas C, Alber M. The incidence of late rectal bleeding in high-dose conformal radiotherapy of prostate cancer using EUD-and Dose-Volume Based NTCP models. Int J Radiat Oncol Biol Phys. 2007;67:1066

21. Semenenko V, Li X. Lyman-Kutcher-Burman NTCP model parameters for radiation pneumonitis and xerostomia based on combined analysis of published clinical data. Phys Med Biol. 2008;53:737.

22. Bakhshandeh M, Hashemi B, Mahdavi SRM, Nikoofar A, Vasheghani M, Kazemnejad A. Normal tissue complication probability modeling of radiation-induced hypothyroidism after head-and-neck radiation therapy. Int J Radiat Oncol Biol Phys. 2013;85:514-21

23. Rancati T, Fiorino C, Sanguineti G. NTCP modeling of subacute/late laryngeal edema scored by fiberoptic examination. Int J Radiat Oncol Biol Phys. 2009;75:915-23.

24. Tucker SL, Dong L, Cheung R, Johnson J, Mohan R, Huang EH, Liu HH, Thames HD, Kuban D: Comparison of rectal dose-wall histogram versus dose-volume histogram for modeling the incidence of late rectal bleeding after radiotherapy. Int J Radiat Oncol Biol Phys 2004, 60:1589-1601.

25. Morgan BJ. Analysis of quantal response data. CRC Press; 1992.

26. Vergouwe $Y$, Moons KG, Steyerberg EW. External validity of risk models: use of benchmark values to disentangle a case-mix effect from incorrect coefficients. Am J Epidemiol. 2010;172:971-80.

27. Steyerberg EW, Vickers AJ, Cook NR, Gerds T, Gonen M, Obuchowski N, et al. Assessing the performance of prediction models: a framework for some traditional and novel measures. Epidemiol (Cambridge, Mass). 2010;21:128.

28. Lee T-F, Chao P-J, Chang L, Ting H-M, Huang Y-J. Developing multivariable normal tissue complication probability model to predict the incidence of symptomatic radiation pneumonitis among breast cancer patients. PLoS One. 2015;10:e0131736.

29. Beetz I, Schilstra C, van der Schaaf A, van den Heuvel ER, Doornaert P, van Luijk $P$, et al. NTCP models for patient-rated xerostomia and sticky saliva after treatment with intensity modulated radiotherapy for head and neck cancer: the role of dosimetric and clinical factors. Radiother Oncol. 2012;105:101-6.

30. Burnham KP, Anderson DR, Huyvaert KP. AIC model selection and multimodel inference in behavioral ecology: some background, observations, and comparisons. Behav Ecol Sociobiol. 2011;65:23-35.

31. Burnham KP, Anderson DR. Multimodel inference understanding AIC and BIC in model selection. Socio Meth Res. 2004;33:261-304.

32. Anderson DR, Burnham KP. Avoiding pitfalls when using information-theoretic methods. J Wildl Manage. 2002;912-918.

33. Bentzen SM, Constine LS, Deasy JO, Eisbruch A, Jackson A, Marks LB, et al. Quantitative Analyses of Normal Tissue Effects in the Clinic (QUANTEC): an introduction to the scientific issues. Int J Radiat Oncol Biol Phys. 2010;76:S3-9.

34. Bhandare N, Jackson A, Eisbruch A, Pan CC, Flickinger JC, Antonelli P, et al. Radiation therapy and hearing loss. Int J Radiat Oncol Biol Phys. 2010;76:S50

35. Hirsch A, Noren G. Audiological findings after stereotactic radiosurgery in acoustic neurinomas. Acta Otolaryngol. 1988;106:244-51.

36. Mujica-Mota M, Waissbluth S, Daniel SJ. Characteristics of radiation-induced sensorineural hearing loss in head and neck cancer: A systematic review. Head Neck. 2013;35:1662-8.

37. Klem ML, Mechalakos JG, Wolden SL, Zelefsky MJ, Singh B, Kraus D, et al. Intensity-modulated radiotherapy for head and neck cancer of unknown primary: toxicity and preliminary efficacy. Int J Radiat Oncol Biol Phys. 2008:70:1100-7.

38. Chen WC, Jackson A, Budnick AS, Pfister DG, Kraus DH, Hunt MA, et al. Sensorineural hearing loss in combined modality treatment of nasopharyngeal carcinoma. Cancer. 2006;106:820-9.

\section{Submit your next manuscript to BioMed Central and take full advantage of:}

- Convenient online submission

- Thorough peer review

- No space constraints or color figure charges

- Immediate publication on acceptance

- Inclusion in PubMed, CAS, Scopus and Google Scholar

- Research which is freely available for redistribution

Submit your manuscript at www.biomedcentral.com/submit
C BioMed Central 